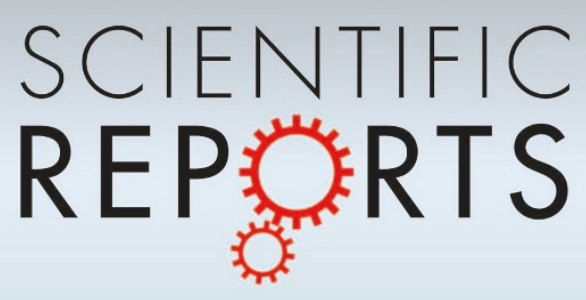

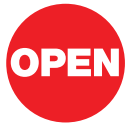

SUBJECT AREAS:

ELECTRICAL AND

ELECTRONIC

ENGINEERING

ELECTRONIC DEVICES

APPLIED PHYSICS

ELECTRONIC PROPERTIES AND MATERIALS

Received

31 October 2012

Accepted

22 January 2013

Published

18 February 2013

Correspondence and requests for materials should be addressed to Z.Q.M. (mazq@engr. wisc.edu)

* These authors contributed equally to this work.

\section{Fast flexible electronics with strained} silicon nanomembranes

\author{
Han Zhou ${ }^{*}$, Jung-Hun Seo ${ }^{*}$, Deborah M. Paskiewicz ${ }^{2 *}$, Ye Zhu $^{2}$, George K. Celler ${ }^{3}$, Paul M. Voyles², \\ Weidong Zhou ${ }^{4}$, Max G. Lagally² \& Zhenqiang Ma'
}

\begin{abstract}
'Department of Electrical and Computer Engineering, University of Wisconsin-Madison, Madison, WI 53706, USA, ${ }^{2}$ Department of Materials Science and Engineering, University of Wisconsin-Madison, Madison, WI 53706, USA, ${ }^{3}$ Institute for Advanced Materials, Devices, and Nanotechnology (IAMDN), and Department of Materials Science and Engineering, Rutgers University, Piscataway, NJ 08854, USA, ${ }^{4}$ Department of Electrical Engineering, NanoFAB Center, University of Texas at Arlington, Arlington, TX 76019, USA.
\end{abstract}

Fast flexible electronics operating at radio frequencies $(>1 \mathrm{GHz})$ are more attractive than traditional flexible electronics because of their versatile capabilities, dramatic power savings when operating at reduced speed and broader spectrum of applications. Transferrable single-crystalline Si nanomembranes (SiNMs) are preferred to other materials for flexible electronics owing to their unique advantages. Further improvement of Si-based device speed implies significant technical and economic advantages. While the mobility of bulk Si can be enhanced using strain techniques, implementing these techniques into transferrable single-crystalline SiNMs has been challenging and not demonstrated. The past approach presents severe challenges to achieve effective doping and desired material topology. Here we demonstrate the combination of strained- NM-compatible doping techniques with self-sustained-strain sharing by applying a strain-sharing scheme between $\mathrm{Si}$ and $\mathrm{SiGe}$ multiple epitaxial layers, to create strained print-transferrable SiNMs. We demonstrate a new speed record of Si-based flexible electronics without using aggressively scaled critical device dimensions.

- lexible electronics have been mainly addressing electronic applications operating at low or moderate speed ${ }^{1-3}$. For these applications, form factors such as bendability and large area are of more importance than speed. Organic semiconductors ${ }^{4,5}$, and amorphous ${ }^{6}$ or polycrystalline $\mathrm{Si}^{7}$, which can be processed at relatively low temperature and with low cost, often suffice to address them. On the other hand, there is a wider spectrum of electronics applications where higher speed and mechanical flexibility are simultaneously needed, such as high-speed and wireless communications, remote sensing and airborne/space surveillance ${ }^{8}$. We entitle the special category of such flexible electronics as fast flexible electronics. A number of applications even require the operating speed (frequency) be beyond $1 \mathrm{GHz}$ and hence these applications can be further termed as radio frequency $(R F)$ flexible electronics. Fast flexible electronics provide superior performance and application advantages. As is known, high-speed devices consume much less power if they are operated at a reduced speed $^{9-11,25}$, which dramatically benefits battery powered devices. Wirelessly connected devices enabled only by high operation speed/frequency are more convenient to use than wired devices ${ }^{1}$ and a high-frequency wireless system is also generally more compact than a low-frequency one.

Transferrable mono-crystalline Si nanomembranes (NMs) are suitable for active material of fast flexible electronics when comparing with any other materials for flexible electronics owing to their material uniformity ${ }^{12}$, mechanical flexibility ${ }^{13}$ and durability ${ }^{14}$, electrical properties equivalent to their bulk counterparts ${ }^{15}$, easy handling ${ }^{16-18}$ and processing, and low cost. Si has modest mobility values in comparison to most III-V and other materials ${ }^{19,20}$, but can be easily down scaled for performance improvement. As large critical device dimensions are generally preferred for flexible-electronics applications where cost and large area are often of the most concern, improving device speed using approaches other than dimension downscaling is preferred. Among them, strain engineering is one of the most effective ones ${ }^{21,22}$. In contrast to bulk $\mathrm{Si}$, where strain in the active device layer can be easily sustained/held by a rigid substrate ${ }^{23}$, in transferrable Si nanomembranes strain needs to be self-sustained. Here we describe a combined strain compatible effective doping and strain engineering approach that is especially suited for transferrable Si NMs. Strain compatible device fabrication techniques then lead us to desired higher device speed on flexible substrates. 


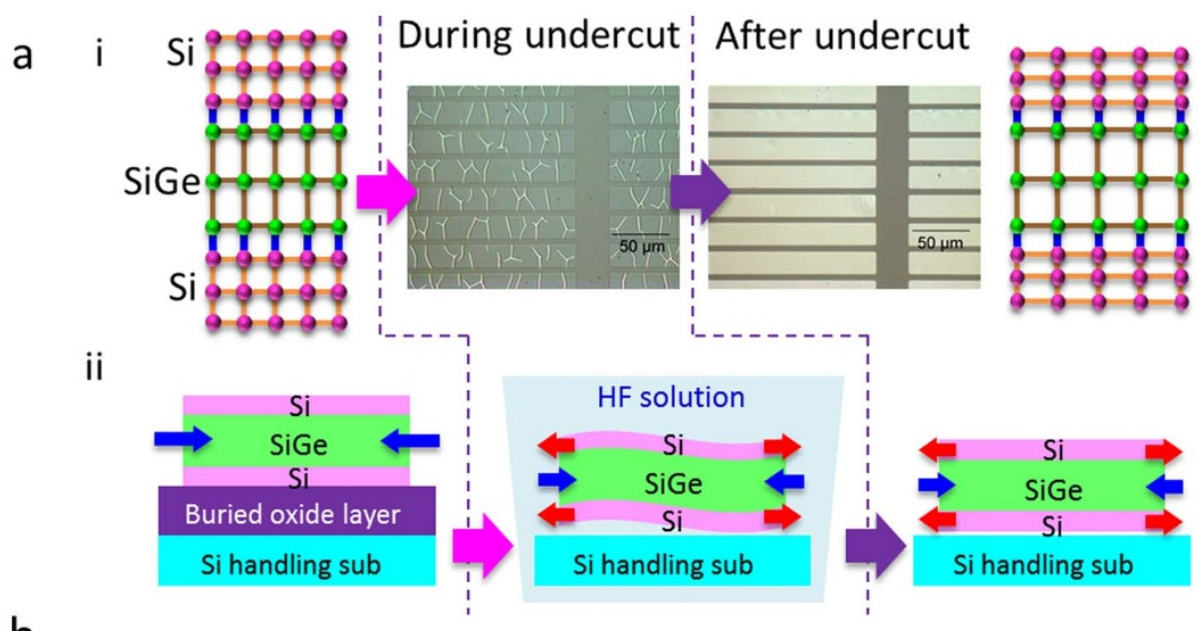

b
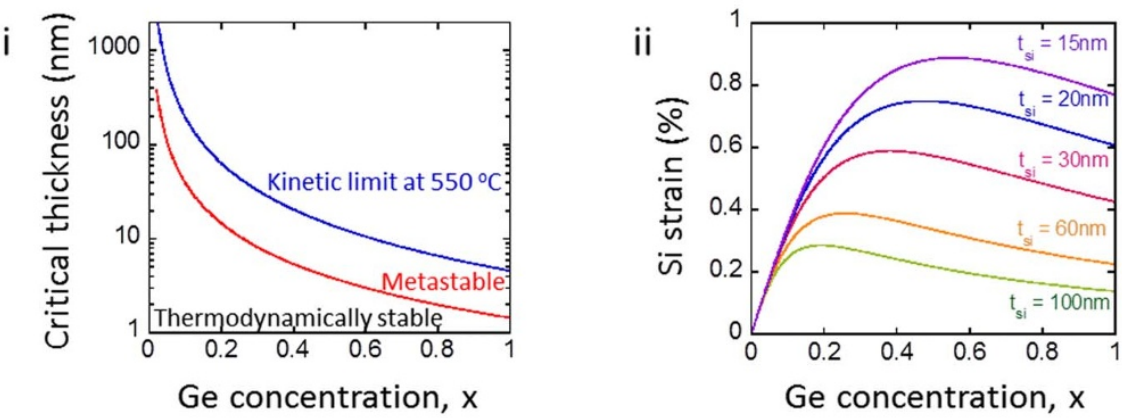

Ge concentration, $x$
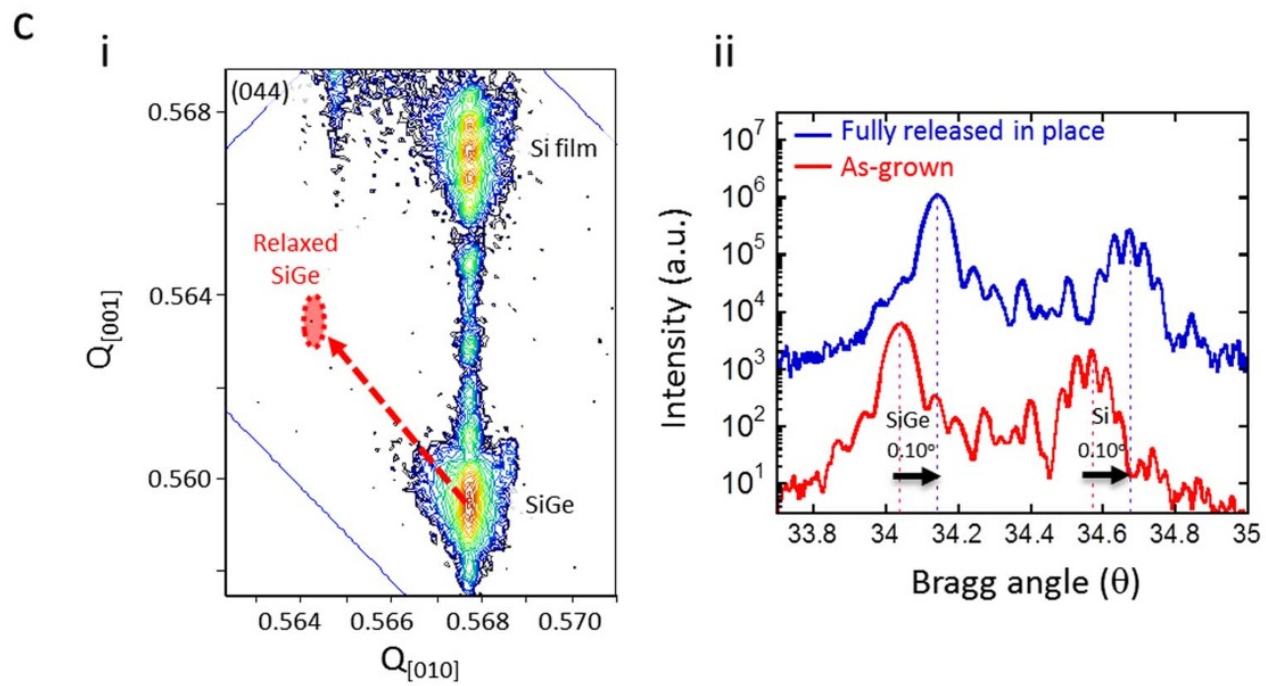

Figure 1 | Strain sharing using Si/SiGe/Si epitaxial trilayer structure. (a), i, Atomic lattice schematic diagram showing the strain sharing principle. Optical images show the strained NM during release and after finishing release. ii, Process flow to implement the strain sharing principle. (b), i, Critical and metastable thicknesses of SiGe that can be coherently grown on Si. ii, Si strain as a function of Ge fraction in SiGe used to guide the design and epitaxial growth of Si/SiGe/Si trilayer. (c), X-ray diffraction measurement verifying strain sharing. i, On-axis line scan around the (004) reflection before and after release of the trilayer NM. ii, Off-axis reciprocal-space map (RSM) around the (044) reflection for the as-grown trilayer structure.

\section{Results}

Figure 1a illustrates the previously developed ${ }^{21,24}$ strain sharing techniques used to create self-sustained strain in Si NMs. First, $80 \mathrm{~nm}$ of undoped $\mathrm{Si}_{0.795} \mathrm{Ge}_{0.205}$ is epitaxially grown on silicon-on-insulator (SOI) with a $48 \mathrm{~nm} \mathrm{Si} \mathrm{template} \mathrm{layer.} \mathrm{A} \mathrm{nearly} \mathrm{symmetrical} \mathrm{trilayer}$ structure $(\mathrm{Si} / \mathrm{SiGe} / \mathrm{Si})$ is formed by growing $46 \mathrm{~nm}$ of undoped $\mathrm{Si}$ on top of the SiGe. Because of the lattice mismatch between Si and SiGe, the SiGe layer in the trilayer structure is compressively strained to the $\mathrm{Si}$ in-plane lattice constant (mismatch strain, $\varepsilon_{\mathrm{m}}=-0.77 \%$ for $\mathrm{Si}_{0.795} \mathrm{Ge}_{0.205}$ ). The trilayer NM is released by selective removal of the
$\mathrm{SiO}_{2}$ (BOX) layer of the SOI. During the release, strain sharing occurs between the SiGe and Si layers; some of the compressive strain in the SiGe layer transfers as tensile strain to the outer Si layers. It is important to have a balanced trilayer structure (top and bottom layers approximately equal thickness) to prevent the heterostructure from curling during release from the handling substrate ${ }^{26}$. Of more importance, the tensile strain in Si layers is self-sustained by a balance of forces between the $\mathrm{Si}$ and $\mathrm{SiGe}$ layers in the freestanding trilayer $\mathrm{NM}^{21,24}$.

The amount of strain transferred to the Si layers is determined by the $\mathrm{Si} / \mathrm{SiGe}$ thickness ratio and Ge composition of the alloy layer, 
which controls the mismatch strain $\left(\varepsilon_{\mathrm{m}}\right)$ in the trilayer ${ }^{24}$. The thickness ratio determines the fractional amount of the mismatch strain in the SiGe alloy that is transferred to the Si layers. The magnitude of strain sharing between the layers increases with increasing SiGe thickness and decreasing Si thickness. The thickness of the SiGe, however, must be kept below the kinetic critical thickness for dislocation formation (Figure $1 \mathrm{~b})^{27}$ because in the as-grown state, the SiGe layer accommodates all the strain in the trilayer. Note that the kinetic critical thickness is different from (i.e., larger than) the thermodynamic critical thickness, because of kinetic barriers to dislocation formation. As the growth temperature is lowered the kinetic critical thickness increases. Thinning the Si in SOI mentioned above allows maximum strain sharing. Figure $1 \mathrm{~b}$ shows the expected strain transfer to the Si layers as a function of Ge composition in the alloy layer and total Si layer thickness $\left(t_{\mathrm{Si}}=\right.$ thickness of top + bottom layers), assuming the alloy layer thickness is equal to the kinetic critical thickness for growth at $550^{\circ} \mathrm{C}$. From knowledge of the layer thicknesses and Ge composition of the initial heterostructure, we expect $\sim 0.35 \%$ biaxial tensile strain in the Si layers after strain sharing. Mobility enhancement is expected from the tensilely strained $\mathrm{Si}^{28}$.

To demonstrate the effectiveness of the self-sustained straining approach, Figure 1c shows an $\mathrm{x}$-ray diffraction (XRD) off-axis reciprocal-space map (RSM) around the (044) reflection for the as-grown trilayer structure. The RSM indicates that the SiGe layer is strained to the Si lattice constant; the $\mathrm{Si}$ and SiGe peaks lie along the same vertical line. This result confirms that there is no plastic relaxation of the mismatch strain in the alloy before release of the trilayer ( $\mathrm{SiGe}$ layer thickness is below kinetic critical thickness for dislocation formation). Figure $1 \mathrm{c}$ also illustrates the strain sharing results in the $\mathrm{Si} / \mathrm{SiGe} / \mathrm{Si}$ layer (an undoped sample is shown). On-axis XRD lines scans around the (004) reflection allow us to measure the outof-plane lattice constant change that occurs during strain sharing. In the as-grown state the SiGe layer is compressively strained to the $\mathrm{Si}$ lattice constant; an in-plane compressive strain translates to an outof-plane expansion (smaller Bragg angle). After release, the SiGe becomes less compressively strained and the Si layers become tensile strained. The expansion in the in-plane lattice constant leads to a reduction in the out-of-plane lattice constant of both layers. Both the $\mathrm{SiGe}$ and $\mathrm{Si}$ peaks shift to higher Bragg angles ( +0.1 degree), indicating that elastic strain sharing occurs between SiGe and Si after release; the relaxation of compressive strain in the SiGe layer is equal to the tensile strain transferred to the Si layers. The strain in the trilayer NM is now self sustained in the released and freestanding NMs.

To realize high device speed and RF operation of devices, the NMs must be doped. The above discussion and all prior work that uses self-sustained strain relate to undoped NMs. When we attempted to dope the strained trilayer NM prior to release, using the doping approach that has been successfully applied to unstrained $\mathrm{Si}$ $\mathrm{NMs}^{29,30}$, the strained $\mathrm{NM}$ curled upon release and became very rough. Figure $2 \mathrm{a}$ briefly illustrates the application of the NM prerelease selective ion implantation doping approach, but being applied to the strained trilayer NM. A detailed doping process flow for unstrained Si NMs is shown in Figure S1. Figure 2b shows a 3D microscopic image of a doped and annealed trilayer NM after release from the handling substrate. As can be seen, the topology of the released trilayer NMs becomes unsuitable for further processing, such as transfer and patterning. We believe that the $\mathrm{Si} / \mathrm{SiGe} / \mathrm{Si}$ epilayer structure has been damaged through atomic mixing and crystalline defects created by the implanted ions. The subsequent annealing procedure intended for recrystallization failed to restore the ion implanted damaged layers and interfaces back to their original order. Instead, significant and unbalanced stress was built in the NM after performing the doping processes, which caused severe curling of the released NMs.
To realize effective doping while maintaining a flat topology of the flexible, strain-shared trilayer NM for fabrication of RF devices, we designed an alternative approach to strain-compatible effective doping processes (Figure 2c). Instead of applying the ion implantation and anneal processes directly on a trilayer NM, we first applied them to an unstrained Si layer on SOI. Typically, low-energy phosphorus ion implantation is used to heavily dope the top portion of a $\mathrm{Si}$ template layer, leaving the bottom portion barely damaged by the implanted ions. An annealing process follows to recrystallize the damage and simultaneously drive the implanted dopants to diffuse until they reach the bottom surface of the template layer, generating a high-level and nearly uniform doping profile across the entire Si template layer. Leaving the bottom portion of the template layer undamaged during implantation is critical, as the bottom portion serves as the seed layer for recrystallization of the entire layer during the annealing process. Detail of ion implantation conditions and results can be found in the Method Section and Figure S2. After finishing these processes, very low sheet resistance is obtained, which is needed to achieve high-frequency device operation (see the Method Section). For unstrained-device fabrication, used as reference devices in this work, the selectively doped Si template layer is ready for release and transfer to a flexible substrate.

To realize selectively doped trilayer Si NMs for RF device fabrication, the selectively doped unstrained Si template layer was thinned down followed by epitaxial growth of $\mathrm{SiGe}$ and $\mathrm{Si}$ layers as performed in the undoped trilayer NM case. Figure 2(d) shows images of trilayer NMs (in the form of strips) at the different stages of processing. The doped regions for the source/drain/source, with $1.5 \mu \mathrm{m}$ gaps between them (a two-gate finger device), are clearly identifiable. It is noted that after finishing the growth, only the bottom Si layer is heavily and selectively doped, with little dopants diffusing back to the $\mathrm{SiGe}$ or to the top Si layers. The doping and thinning down procedures have some effect on the crystallinity of the as-grown $\mathrm{Si} / \mathrm{SiGe} / \mathrm{Si}$ trilayers (as indicated by a general broadening of the diffraction peaks in Figure 2e(ii)). The SiGe layer, however, is still strained to the Si lattice constant so strain sharing is expected to occur as predicted upon release of these trilayers $\left(\varepsilon_{\mathrm{Si}}=0.34 \% \pm 0.01 \%\right)$. With this amount of biaxial tensile strain in $\mathrm{Si}$, we can expect a $\sim 47 \%$ increase in the electron mobility ${ }^{28}$.

Figure 3a provides the illustration of the device (field effect thinfilm transistor, TFT) fabrication procedures using the transferred trilayer NM, which is bonded to a polyethylene terephthalate (PET) substrate. Using a flip transfer procedure, the selectively doped source and drain regions on the bottom Si layer of the trilayer NM becomes the top surface. Following the procedures described previously $^{31}$, the device fabrication is finished. Figures $3 b$ and $3 c$ show the cross section, dimensions, and optical image of a finished device on a PET substrate. Figures $3 \mathrm{~d}$ and $3 \mathrm{e}$ show the reference device fabrication and information on the unstrained Si NM. The gate length and channel length, which are identically applied to both the strained and the unstrained NM, are 2.5 and $1.5 \mu \mathrm{m}$, respectively.

Figure $3 \mathrm{f}(\mathrm{i})$ shows the transfer curve and calculated transconductance $\left(g_{\mathrm{m}}\right)$ versus gate voltage $\left(\mathrm{V}_{\mathrm{g}}\right)$ of a strained-channel TFT along with the results measured from the unstrained-channel TFT for comparison. The highest $\mathrm{g}_{\mathrm{m}}$ values for the strained and the unstrained TFTs are $386 \mu \mathrm{S}$ and $262 \mu \mathrm{S}$, respectively. Because the dimensions of the two devices are identical, the $47.3 \%$ enhancement of the peak $g_{\mathrm{m}}$ values in the strained TFT is mainly ascribed to mobility enhancement, which is caused by the introduction of the tensile strain in the Si channel of the trilayer. The $g_{m}$ and mobility enhancement ratios are consistent with the expected values ${ }^{28}$. Figure $3 \mathrm{f}(\mathrm{ii})$ plots $\mathrm{g}_{\mathrm{m}}$ versus drain current. The roughly linear trend of $g_{m}$ as a function of logarithmic drain current is consistent with devices made on rigid substrates ${ }^{9-11}$. Figure $3 f(i i i)$ shows the measured current gain $\left(\mathrm{H}_{21}\right)$ and power gain $\left(\mathrm{G}_{\max }\right)$ of the strained TFT, indicating that the cut-off frequency $\left(f_{\mathrm{T}}\right)$ is $5.1 \mathrm{GHz}$ and the 
a

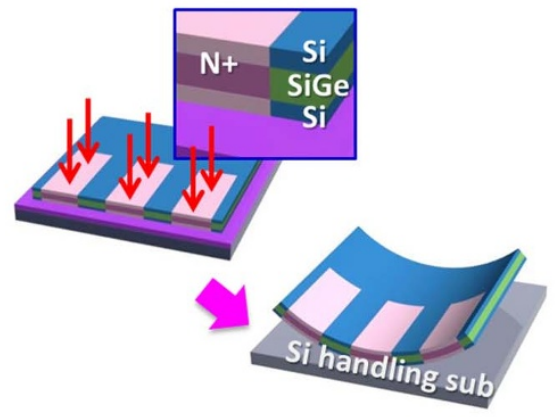

b i

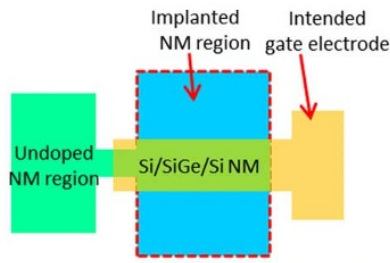

ii

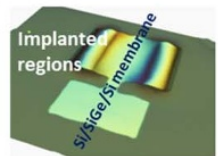

(a) 3D microscopic image

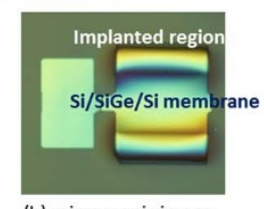

(b) microscopic image

C
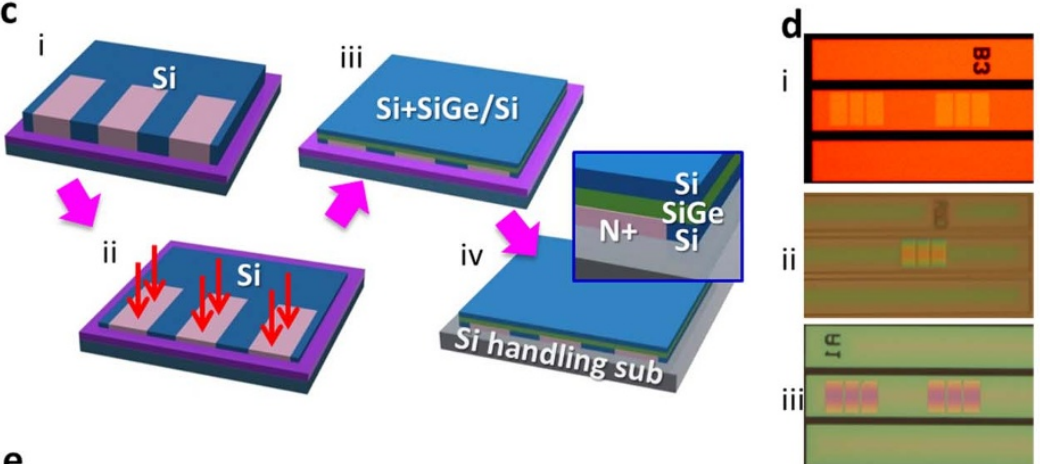

e

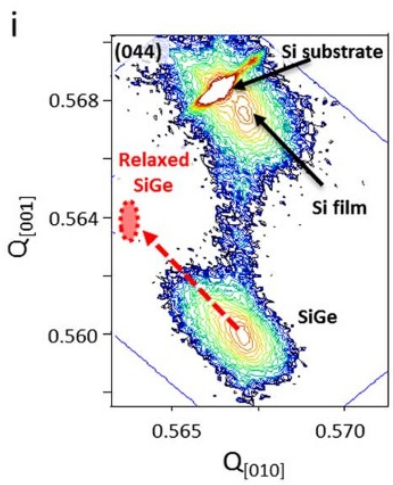

ii

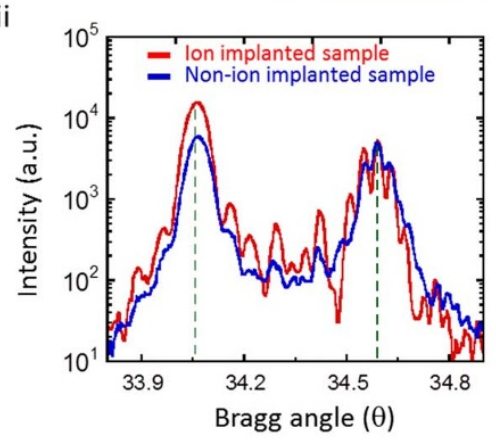

Figure $2 \mid$ Doping of strain shared $\mathrm{Si} / \mathrm{SiGe} / \mathrm{Si}$ trilayer nanomembrane. (a), Ion implantation and anneal processes are applied to as-grown $\mathrm{Si} / \mathrm{SiGe} / \mathrm{Si}$ trilayer. (b), i, Schematic of patterned strained NM for RF device fabrication. ii, 3D microscopic images of released strained trilayer, showing the un-flat topology. Note that the undoped region of the trilayer NM stays flat. (c), Doping of strained trilayer structure. i, Unstrained Si NM is ion implanted and annealed. ii, Si NM is thinned down. iii, SiGe and Si epi growth on thinned Si NM to form symmetric trilayer. iv, Release of Si/SiGe/Si trilayer. (d), Images of doped strained NM at different processing stages. i. Before release. ii. After finishing undercut, sitting on Si handling substrate. iii, After being transferred to a plastic substrate. (e), X-ray diffraction of as-grown ion-implanted trilayer NM. i. Off-axis reciprocal-space map (RSM) around the (044) reflection. ii. On-axis line scans around the (004) reflection comparing the doped and undoped as-grown trilayer NMs.

maximum oscillation frequency $\left(f_{\max }\right)$ is $15.1 \mathrm{GHz}$. For RF applications (analog circuits), power gain is more significant than current gain, which is typically used for evaluating switching speed of (digital/logic) devices. As a result, $f_{\max }$ is considered a better indicator of the device's speed than $f_{\mathrm{T}}$. The $f_{\max }$ value sets a new speed record for Si-based TFTs fabricated on plastic substrates, even though critical dimensions of the strained channel are larger than the previously reported devices ${ }^{30}$ and a relatively thick gate dielectric $(120 \mathrm{~nm})$ is used. Considering biomedical wireless devices typically operating at $400 \mathrm{MHz}^{32}$, for which both the strained-channel transistor (this work, on plastic) and poly-Si transistors (on glass) having a $f_{\max }$ of $3.5 \mathrm{GHz}^{33}$ can be used, the strained transistor consumes roughly two orders less power than the poly-Si transistor, as indicated by points $\mathrm{A}$ and $\mathrm{B}$ in Figure $3 \mathrm{f}(\mathrm{ii})$. As a comparison, the unstrained reference TFT with identical dimensions has $f_{\mathrm{T}}$ and $f_{\max }$ of $3.3 \mathrm{GHz}$ and $10.3 \mathrm{GHz}$, respectively. The mobility enhancement $(47.3 \%)$ is directly reflected in the device's speed enhancement: $54.5 \%$ for $f_{\mathrm{T}}$ and $46.6 \%$ for $f_{\max }$. Following the typical scaling law of field effect transistors, if a smaller device feature is applied to the strained-channel devices by using a previously demonstrated alignment scheme ${ }^{30}$, about $6 \mathrm{GHz} f_{\mathrm{T}}$ and $18 \mathrm{GHz} f_{\max }$ can be expected. Figures $3 \mathrm{f}(\mathrm{iv}-\mathrm{v})$ show the gate bias dependence under fixed drain bias and drain bias dependence under fixed gate bias of the frequency response characteristics of the strained-channel TFT, respectively. Overall, relatively low bias voltages and thus low power consumptions are needed to operate these high-speed flexible transistors, a significant advantage of the singlecrystal NM based TFTs over the polycrystalline Si-based TFTs ${ }^{34}$, where much higher operation voltages are generally needed.

Figure 4 shows the mobility and RF characteristics of the strained and the unstrained transistors under bending situations along with the bending test setup and an optical image of a bent array on a PET substrate. Besides the effective mobility enhancement of $47.3 \%$ 
a

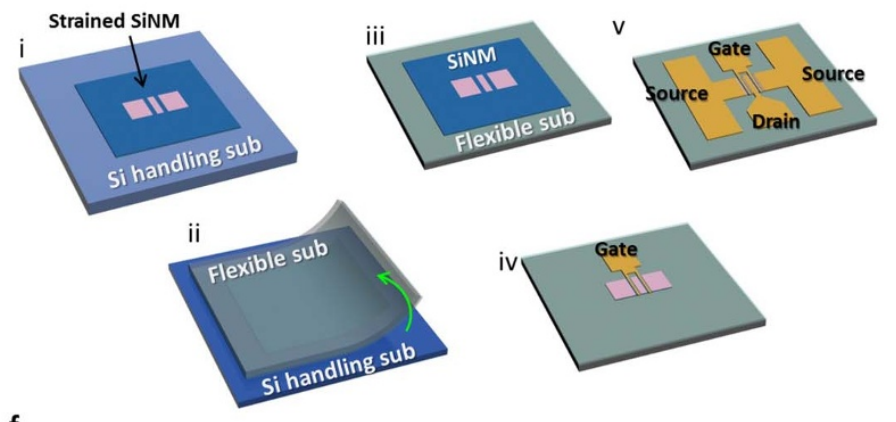

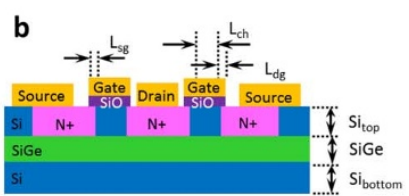

c

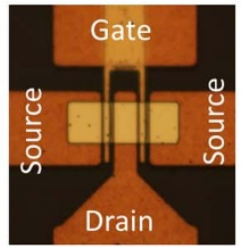

d
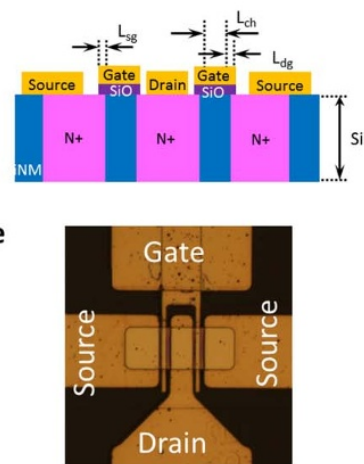

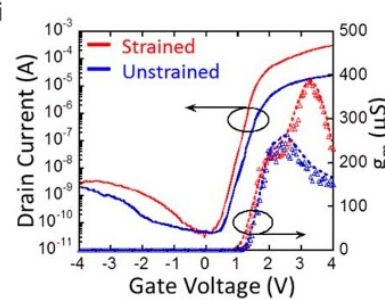

ii

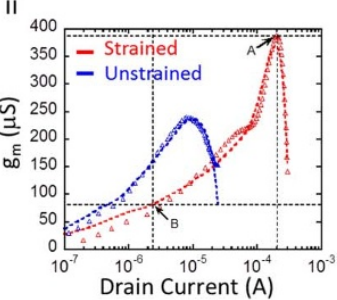

iii

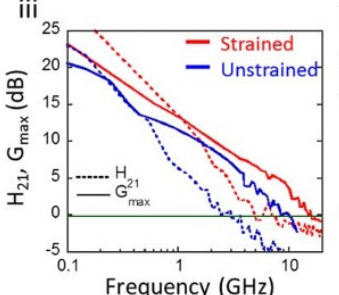

iv

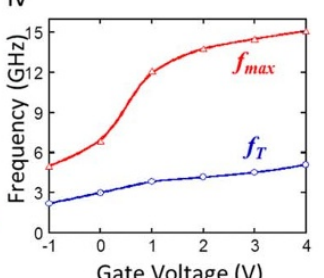

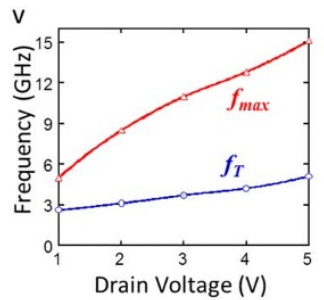

Figure $3 \mid$ Device fabrication on released strained Si/SiGe/Si NMs and characterizations. (a), Process flow for device fabrication. i, Released strained NM sitting on handling substrate. ii-iii, NM is flip transferred to a PET substrate. iv, Gate stack formation using lift-off techniques. v, Source/drain and a interconnect spelling-metallization. (b), Cross sectional illustration of strained-NM device dimensions. (c), Optical image of a fabricated strained device. (d), Cross sectional illustration of unstrained device dimensions. (e), Optical image of a fabricated unstrained device. For both strained and unstrained devices, the gate lengths $\left(\mathrm{L}_{\mathrm{g}}=\mathrm{L}_{\mathrm{ch}}+\mathrm{L}_{\mathrm{sg}}+\mathrm{L}_{\mathrm{dg}}\right)$ is $2.5 \mu \mathrm{m}$ and gate width is $40 \mu \mathrm{m}$. Having a gate overlap distance with source/drain regions $\left(\mathrm{L}_{\mathrm{sg}}\right.$ and $\left.\mathrm{L}_{\mathrm{dg}}\right)$ of $0.5 \mu \mathrm{m}$, the effective channel lengths $\left(\mathrm{L}_{\mathrm{ch}}\right)$ are $1.5 \mu \mathrm{m}$. (f), Device DC and RF response characteristics of unstrained and strained devices. i, Transfer curves and calculated transconductance $\left(\mathrm{g}_{\mathrm{m}}\right)$ curves of unstrained and strained devices $\left(\mathrm{V}_{\mathrm{ds}}=500 \mathrm{mV}\right)$. ii, $\mathrm{g}_{\mathrm{m}}$ is plotted as a function of drain current. Point $\mathrm{A}$ indicates the peak $\mathrm{g}_{\mathrm{m}}$ where peak $f_{\mathrm{T}} / f_{\max }$ values were measured. Point $\mathrm{B}$ is where $3.5 \mathrm{GHz} f_{\max }$ can be obtained. The drain current at point $\mathrm{B}$ is roughly two orders lower than that at point A. iii, Current gain $\left(\mathrm{H}_{21}\right)$ and power gain $\left(\mathrm{G}_{\mathrm{max}}\right)$ as a function of frequency of unstrained and strained devices $\left(\mathrm{V}_{\mathrm{g}}=4 \mathrm{~V}\right.$, $\left.\mathrm{V}_{\mathrm{ds}}=5 \mathrm{~V}\right)$. iv and $\mathrm{v}, f_{\mathrm{T}}$ and $f_{\max }$ of strained devices as a function of gate bias under fixed drain bias $\left(\mathrm{V}_{\mathrm{ds}}=5 \mathrm{~V}\right)$ and that as a function of drain bias under fixed gate bias $\left(\mathrm{V}_{\mathrm{g}}=4 \mathrm{~V}\right)$, respectively.

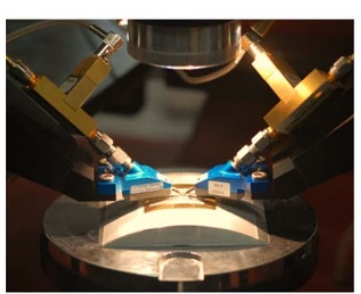

b

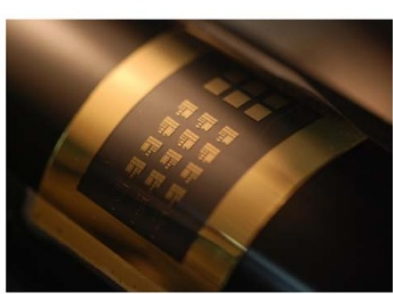

C
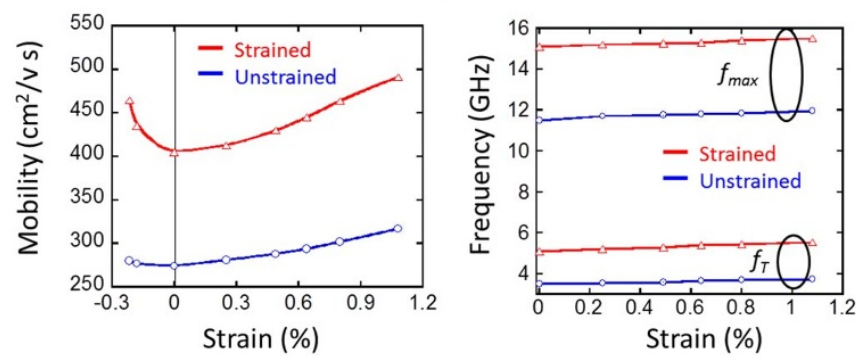

Figure 4 | Device characteristics under bending. (a), Bending setup for RF measurements. (b), A bent device array on a bending fixture.

(c), Calculated mobility values from measured transconductance as a function of bending induced strain for both unstrained and strained devices. (d), $f_{\mathrm{T}}$ and $f_{\text {max }}$ of both unstrained and strained devices as a function of bending induced external strain. without applying any external strain, the mobility in both the strained and the unstrained transistor channels was further enhanced, $20 \%$ and $14.8 \%$, respectively, by applying external uniaxial strain of $1.08 \%$ (measured from bending curvature). A mobility enhancement (13\%) was also observed from the strained channel under a uniaxial externally applied compressive strain of $-0.215 \%$, indicating the possibility to create a complementary strain-shared structure: $\mathrm{SiGe} / \mathrm{Si} / \mathrm{SiGe}$ for higher-performance flexible transistors, where SiGe serves as the device channel. The measured mobility variation trends are consistent with that of strained bulk $\mathrm{Si}^{28}$. The frequency variation trend as a function of tensile strain (impossible to measure frequency response under concave bending due to large RF probe size) is consistent with that of the mobility. It is noted that the transistors remain intact and operational under high-strain conditions; a convex radius of curvature of $15.5 \mathrm{~mm}$ translates into an external strain of $1.08 \%$.

\section{Discussion}

In summary, we have demonstrated a simple and viable approach to realizing strained-mono-crystalline-Si RF transistors on flexible plastic substrates. This technique has great potential in low-power and high-speed flexible-electronics applications, and could be used to replace a number of rigid counterparts for use in mechanically bendable and non-planar conformal surfaces where rigid devices cannot be easily used. One can foresee as a consequence manufacturable large-area applications of such flexible high-speed thin-film transistor technology.

\section{Methods}

Doping of unstrained Si nanomembrane. Effective doping of the SiNM is needed to reduce contact resistance in source and drain regions of RF transistors. Commercially 
available SOI (Soitec USA, 2 Centennial Drive, Peabody, MA 01960, USA) with $200 \mathrm{~nm} \mathrm{Si} \mathrm{(001)} \mathrm{template} \mathrm{and} 145 \mathrm{~nm}$ buried oxide (BOX) layers is used as the starting material. The $\mathrm{Si}(001)$ template is lightly doped with boron. Phosphorus ion implantation is used on the SOI substrate with a dose of $2 \times 10^{15} \mathrm{~cm}^{-2}$ and an energy of $20 \mathrm{keV}$. Following the ion implantation, the sample is annealed in a high-temperature furnace at $950^{\circ} \mathrm{C}$ for 30 mins in $\mathrm{N}_{2}$ ambient to re-crystallize the $\mathrm{Si}$ template and activate the dopants. TEM cross section images of Si NMs (unstrained) before and after anneal are shown in Figure S1. The simulated and characterized doping profiles using secondary ion mass sepectroscopy (SIMS) before and after anneal are shown in Figure S2.

Thinning down Si nanomembrane. After the ion implantation, the Si template is treated in an RIE chamber (Unaxis 790, $30 \mathrm{~W}$ ) with $\mathrm{SF}_{6} / \mathrm{O}_{2}$ for $40 \mathrm{sec}$. A dry thermal oxidation at $1050^{\circ} \mathrm{C}$ for $10 \mathrm{~min}$ is applied to the sample to further reduce the $\mathrm{Si}$ template to the desired thickness in the $\mathrm{Si} / \mathrm{SiGe} / \mathrm{Si}$ tri-layer structure. The final thickness of the Si template is $48 \mathrm{~nm}$, as verified by XRD measurement.

Epitaxial growth of $\mathrm{SiGe} / \mathrm{Si}$ layers. Si/SiGe/Si trilayer NM fabrication starts with molecular beam epitaxy (MBE) growth of thin SiGe alloy films on SOI(001) substrates with a $\sim 48 \mathrm{~nm}$ top Si template layer and $150 \mathrm{~nm}$ buried oxide (BOX) layer. Our standard chemical cleaning procedure before growth on $\mathrm{Si}$ is as follows: [1] $20 \mathrm{sec}$ in $10 \% \mathrm{HF}$, [2] 10 min in Piranha clean $\left(\sim 80^{\circ} \mathrm{C}_{2} \mathrm{SO}_{4}+\mathrm{H}_{2} \mathrm{O}_{2}\right.$ solution $)$, [3] $15 \mathrm{~min}$ in standard clean $1[\mathrm{SC} 1]\left(\sim 80^{\circ} \mathrm{C} \mathrm{H}_{2} \mathrm{O}+\mathrm{NH}_{4} \mathrm{OH}+\mathrm{H}_{2} \mathrm{O}_{2}\right.$ solution), and [4] $20 \mathrm{sec}$ in $10 \% \mathrm{HF}$ (with a 5-min DI water rinse between each step) before putting the sample directly into the high-vacuum growth chamber. We resistively heat the substrate to $475^{\circ} \mathrm{C}$ during pseudomorphic growth of the alloy and used a growth rate $\sim 3 \mathrm{~nm} /$ $\min . \theta / 2 \theta$ lines scans around the (004) reflection (Figure $1 \mathrm{c}$ and $2 \mathrm{e}$ ) were fit to simulations to extract the Ge composition of the SiGe layer and each of the layer thicknesses before release from the initial growth substrate: $46 \mathrm{~nm} \mathrm{Si} / 80 \mathrm{~nm}$ $\mathrm{Si}_{0795} \mathrm{Ge}_{0205} / 48 \mathrm{~nm} \mathrm{Si}$. In the as-grown heterostructure, the main peak at lower Bragg angles is from the SiGe layer and the broad peak modulated by the thickness fringes is from the two Si layers.

Strain calculation of $\mathbf{S i}$ in $\mathbf{S i} / \mathbf{S i G e / S i}$. The Ge composition will determine the mismatch strain, and the thickness ratio controls the fractional amount of that mismatch strain that is transferred to the Si layers. The strain transferred to the Si layers is:

$$
\varepsilon_{S i}=-\frac{1}{1+\frac{M_{S i} \cdot t_{S i}}{M_{S i G e} \cdot t_{S i G e}}} \cdot \varepsilon_{m},
$$

where $M_{i}$ is the biaxial modulus and $t_{i}$ is the thickness of the respective layers. The mismatch strain, $\varepsilon_{m}$, is the amount of strain in the SiGe before release from the handling substrate.

$$
\begin{aligned}
& \varepsilon_{m}=\frac{a_{S i}-a_{S i G e}}{a_{S i G e}}, \\
& a_{S i G e}=\left[5.431+0.2 x+0.027 x^{2}\right] \dot{\mathrm{A}}
\end{aligned}
$$

Device fabrication. After the trilayer growth (for the strained-channel device) and ion implantation (for the unstrained-channel reference device), optical photolithography is used to pattern both types of the active layers into $40 \mu \mathrm{m}$ wide strips, with $10 \mu \mathrm{m}$ gaps between them etched using reactive ion etching (RIE). The photoresist on top is removed in acetone and the strips are released in a $4: 1$ diluted HF ( $49 \% \mathrm{HF})$ solution in which the BOX layer is selectively etched away. The strips fall onto the Si substrate and, during release, the trilayer $\mathrm{Si} / \mathrm{SiGe} / \mathrm{Si}$ structure shares strain elastically, leaving the top and bottom Si layers tensilely strained. Photoresist SU8-2002 (Microchem Corp.) is spun on the PET host substrate and the freestanding, elastically relaxed strips are transferred top side down onto the SU8 layer. To create better adhesion between the strips and SU8 layer, the Si substrate on which the strips had come to rest after release is gently pressed and then peeled off. The PET substrate is cured from the back side under UV light and baked at $105^{\circ} \mathrm{C}$ for $5 \mathrm{~min}$. After this step, the strips are firmly attached to the PET substrate and ready for the subsequent processing. The color difference between doped source/drain regions and channel regions helps alignment of gate patterns. To make gate dielectric and gate metal contacts, $120 \mathrm{~nm}$ thick SiO, $20 \mathrm{~nm}$ thick Ti and $150 \mathrm{~nm}$ Au are evaporated on the channel areas defined by optical photolithography. The source/drain metal consisting of $20 \mathrm{~nm}$ Ti and $300 \mathrm{~nm}$ Au is formed on both types of the active layers by optical photolithography and liftoff.

DC and RF characterizations of devices. DC characteristics were measured with an Agilent 4155 semiconductor parameter analyzer in a dark environment. For RF characteristics, Scattering (S) parameter measurement was taken using an Agilent E8364A network analyzer. The "open" and "short" features were used for a deembedding procedure to obtain the intrinsic RF characteristics of device. The deembedding procedure follows the equation;

$$
Y_{\text {transistor }}=\left[\left(Y_{\text {DUT }}-Y_{\text {open }}\right)^{-1}-\left(Y_{\text {short }}-Y_{\text {open }}\right)^{-1}\right]^{-1} .
$$

The effects of contact resistance $f_{\max }$ can be seen in the following equations:

$$
\begin{aligned}
& f_{T}=\frac{g_{m}}{2 \pi\left(C_{g s}+C_{g d}\right)}, \\
& f_{\max }=\frac{f_{T}}{2 \sqrt{\left(R_{g}+R_{s}\right) \cdot g_{o}+2 \pi \cdot R_{g} \cdot C_{g d} \cdot f_{T}}}
\end{aligned}
$$

where $g_{\mathrm{m}}$ is transconductance, $\mathrm{C}_{\mathrm{gd}}$ and $\mathrm{C}_{\mathrm{gs}}$ are gate-drain and gate-to-source capacitance, respectively. $g_{o}$ is output conductance. $R_{g}$ and $R_{s}$ are gate resistance and source resistance, respectively.

Power savings when operating a device at reduced speed. The relationship between power consumption (estimated only based on drain current, which is conservative) and $f_{\max }$ can be estimated by the following equations ${ }^{11}$ :

- Equation for calculating transconductance;

$$
\begin{aligned}
& I_{D}=\frac{1}{2} \cdot \mu \cdot C_{O X} \cdot \frac{W}{L} \cdot\left(V_{G S}-V_{t h}\right)^{2} \\
& g_{m}=\frac{d I_{D}}{d V_{G S}}=k \cdot\left(V_{G S}-V_{t h}\right) \\
& g_{m} \propto\left(V_{G S}-V_{t h}\right)
\end{aligned}
$$

- Equation for calculating drain current;

$$
\begin{aligned}
& I_{D}=I_{0} \cdot\left(\frac{q}{n k T}\right) \cdot \exp \left(\frac{q\left(V_{G S}-V_{t h}\right)}{n k T}\right) \\
& \log \left(I_{D}\right)=k^{\prime}+\frac{q\left(V_{G S}-V_{t h}\right)}{n k T}
\end{aligned}
$$

- $\log \left(I_{D}\right)$ is proportional to $\mathrm{g}_{\mathrm{m}}$ as follows;

$$
\log \left(I_{D}\right) \approx\left(V_{G S}-V_{t h}\right) \propto g_{m}
$$

- Therefore the relationship between $g_{m}$ and RF response is as follows;

$$
f_{T}=\frac{g_{m}}{2 \pi\left(C_{g^{s}}+C_{g d}\right)} \rightarrow f_{T} \propto \log \left(I_{D}\right)
$$

$$
\begin{aligned}
f_{\max } & =\frac{f_{T}}{2 \sqrt{\left(R_{g}+R_{s}\right) \cdot g_{o}+2 \pi \cdot R_{g} \cdot C_{g d} \cdot f_{T}}} \\
& \approx \frac{f_{T}}{2 \sqrt{\left(R_{g}+R_{s}\right) \cdot g_{o}}} \rightarrow f_{\max } \propto \log \left(I_{D}\right)
\end{aligned}
$$

By plotting $g_{\mathrm{m}}$ versus $\log \left(I_{D}\right)$, power consumption at lower $f_{\max }$ can be estimated.

1. Kim, D.-H. et al. Epidermal Electronics. Science 333, 838-843 (2011).

2. Kim, D.-H. et al. Materials and noncoplanar mesh designs for integrated circuits with linear elastic responses to extreme mechanical deformations. Proc. Natl Acad. Sci. USA 105, 18675-18680 (2008).

3. Ahn, J.-H. et al. Bendable integrated circuits on plastic substrates by use of printed ribbons of single-crystalline silicon. Appl. Phys. Lett. 90, 213501 (2007).

4. Forrest, S. R. The path to ubiquitous and low-cost organic electronic appliances on plastic. Nature 428, 911-918 (2004)

5. Gelinck, G. H. et al. Flexible active-matrix displays and shift registers based on solution-processed organic transistors. Nat. Mater. 3, 106-110 (2004).

6. Gleskova, H., Wagner, S., Gašparík, V. \& Kováč, P. $150^{\circ} \mathrm{C}$ Amorphous Silicon Thin-Film Transistor Technology for Polyimide Substrates. J. Electrochem. Soc. 148, G370-G374 (2001).

7. Carey, P. G., Smith, P. M., Theiss, S. D. \& Wickboldt, P. Polysilicon thin film transistors fabricated on low temperature plastic substrates. J. Vac. Sci. Technol. A17, 1946-1949 (1999).

8. Reuss, R. H. et al. Macroelectronics: Perspectives on Technology and Applications. Proc. IEEE 93, 1239-1256 (2005).

9. Terry, Y. et al. Algorithmic Design of CMOS LNAs and PAs for $60-\mathrm{GHz}$ Radio IEEE J. Solid-State Circuits 42, 1044-1057 (2007).

10. Taur, Y. \& Ning, H. T. Fundamentals of Modern VLSI Devices (Cambridge Univ. Press, New York, 1988)

11. Voinigescu, S. \& Dickson, T. High-Frequency Integrated Circuits (Cambridge Univ. Press, New York, available in 2013).

12. Menard, E., Nuzzo, R. G. \& Rogers, J. A. Bendable single crystal silicon thin film transistors formed by printing on plastic substrates. Appl. Phys. Lett. 86, 093507 (2005).

13. Sun, Y., Choi, W. M., Jiang, H., Huang, Y. Y. \& Rogers, J. A. Controlled buckling of semiconductor nanoribbons for stretchable electronics. Nat. Nano. 1, 201-207 (2006).

14. Zhu, Z. T., Menard, E., Hurley, K., Nuzzo, R. G. \& Rogers, J. A. Spin on dopants for high-performance single-crystal silicon transistors on flexible plastic substrates. Appl. Phys. Lett. 86, 133507 (2005).

15. Ahn, J.-H. et al. High-speed mechanically flexible single-crystal silicon thin-film transistors on plastic substrates. IEEE Electron Device Lett. 27, 460-462 (2006). 
16. Meitl, M. A. et al. Transfer printing by kinetic control of adhesion to an elastomeric stamp. Nat. Mater. 5, 33-38 (2006).

17. Kim, S. et al. Microstructured elastomeric surfaces with reversible adhesion and examples of their use in deterministic assembly by transfer printing. Proc. Natl Acad. Sci. USA 107, 17095-17100 (2010).

18. Li, R. et al. Axisymmetric thermo-mechanical analysis of laser-driven non-contact transfer printing. Int. J. Fract. 176, 189-194 (2012).

19. Bolotin, K. I. et al. Ultrahigh electron mobility in suspended graphene. Solid State Commun. 146, 351-355 (2008).

20. Dürkop, T., Getty, S. A., Cobas, E. \& Fuhrer, M. S. Extraordinary Mobility in Semiconducting Carbon Nanotubes. Nano Lett. 4, 35-39 (2003).

21. Yuan, H.-C., Ma, Z., Roberts, M. M., Savage, D. E. \& Lagally, M. G. High-speed strained-single-crystal-silicon thin-film transistors on flexible polymers. J. Appl. Phys. 100, 013708 (2006).

22. Scott, A. S. \& Lagally, G. M. Elastically strain-sharing nanomembranes: flexible and transferable strained silicon and silicon-germanium alloys. J. Phys. D: Appl. Phys. 40, R75 (2007)

23. Thompson, S. E. et al. A 90-nm logic technology featuring strained-silicon. IEEE Trans. Electron Devices 51, 1790-1797 (2004).

24. Roberts, M. M. et al. Elastically relaxed free-standing strained-silicon nanomembranes. Nat. Mater. 5, 388-393 (2006).

25. ITRS 2011 Technology Working Group Reports - Process Integration, Devices, and Structures (PIDS), http://www.itrs.net/Links/2011ITRS/Home2011.htm (2011).

26. Huang, M. et al. Nanomechanical Architecture of Strained Bilayer Thin Films: From Design Principles to Experimental Fabrication. Adv. Mater. 17, 2860-2864 (2005).

27. Houghton, D. C. Strain relaxation kinetics in $\mathrm{Si}_{1-\mathrm{x}} \mathrm{Ge}_{\mathrm{x}} / \mathrm{Si}$ heterostructures. J. Appl. Phys. 70, 2136-2151 (1991).

28. Rim, K. et al. Fabrication and mobility characteristics of ultra-thin strained $\mathrm{Si}$ directly on insulator (SSDOI) MOSFETs Electron Devices Meeting, 2003. IEDM '03 Technical Digest. IEEE International. 2003.12.8-2003.12.10.

29. Yuan, H.-C. \& Ma, Z. Microwave thin-film transistors using Si nanomembranes on flexible polymer substrate. Applied Physics Letters 89, 212105 (2006).

30. Sun, L. et al. Flexible electronics: $12-\mathrm{GHz}$ Thin-Film Transistors on Transferrable Silicon Nanomembranes for High-Performance Flexible Electronics. Small 6 2473 (2010).
31. Zhang, K., Seo, J.-H, Zhou, W. \& Ma, Z. Fast flexible electronics using transferrable silicon nanomembranes. J. Phys. D: Appl. Phys. 45, 143001 (2012).

32. Kwak, K. S., Ullah, S. \& Ullah, N. An Overview of IEEE 802.15.6 Standard. Applied Sciences in Biomedical and Communication Technologies (ISABEL), $20103 \mathrm{rd}$ International Symposium on. 2010.11.7-2010.11.10.

33. Chen, Y.-J. E., Lee, Y.-J. \& Yu, Y.-H. Investigation of Polysilicon Thin-Film Transistor Technology for RF Applications. IEEE Trans. Microw. Theory Techn. 58, 3444-3451 (2010)

34. Qin, G., Yuan, H.-C., Yang, H., Zhou, W. \& Ma, Z. High-performance flexible thin-film transistors fabricated using print-transferrable polycrystalline silicon membranes on a plastic substrate. Semicond. Sci. Technol. 26, 025005 (2011).

\section{Acknowledgments}

This work was supported by AFOSR MURI under grant FA9550-08-1-0337. The program manager at AFOSR is Dr. Gernot Pomrenke. Maintenance support for the SiGe molecular beam epitaxy facility was provided by DOE, Grant DE-FG02-03ER46028, as part of the SiNM synthesis and processing effort. D. M. P. acknowledges support from the NSF Graduate Research Fellowship Program.

\section{Author contributions}

H.Z., J.-H.S., D.M.P., Y.Z, G.K.C., P.M.V., W.Z., M.G.L. and Z.M. performed research; Z.M. designed research; H.Z., J.-H.S., D.M.P., M.G.L. and Z.M. analyzed data; and H.Z., J.-H.S., D.M.P., M.G.L. and Z.M. wrote the paper.

\section{Additional information}

Supplementary information accompanies this paper at http://www.nature.com/ scientificreports

Competing financial interests: The authors declare no competing financial interests.

License: This work is licensed under a Creative Commons

Attribution-NonCommercial-NoDerivs 3.0 Unported License. To view a copy of this license, visit http://creativecommons.org/licenses/by-nc-nd/3.0/

How to cite this article: Zhou, H. et al. Fast flexible electronics with strained silicon nanomembranes. Sci. Rep. 3, 1291; DOI:10.1038/srep01291 (2013). 